\title{
Salt-induced root protein profile changes in seedlings of maize inbred lines with differing salt tolerances
}

\author{
Yujing Cheng', Guoqing Chen ${ }^{1}$, Derong Hao' ${ }^{1}$ Huhua Lu ${ }^{1}$, Mingliang Shi ${ }^{1}$, Yuxiang Mao', \\ Xiaolan Huang ${ }^{1}$, Zhenliang Zhang ${ }^{1}$, and Lin Xue ${ }^{{ }^{*}}$
}

Salt stress is one of the severest growth limited-factors to agriculture production. To gain in-depth knowledge of salt-stress response mechanisms, the proteomics analysis from two maize (Zea mays L.) inbred lines was carried out using twodimensional gel electrophoresis (2-DGE) and matrix-assisted laser desorption/ionization time-of-flight mass spectrometry (MALDI-TOF/TOF-MS). There were 57 salt-regulated proteins identified, 21 and 36 proteins were differentially regulated in inbred lines 'Nongda 1145' (salt-resistant) and 'D340' (salt-sensitive), respectively. The identified proteins were distributed in 11 biological processes and seven molecular functions. Under salt stress, proteins related to antioxidation and lignin synthesis were increased in both inbred lines. The relative abundance of proteins involved in translation initiation, elongation, and protein proteolysis increased in 'Nongda 1145' and decreased in 'D340'. In addition, the abundance of proteins involved in carbohydrate metabolism, protein refolding, ATP synthase and transcription differed between the two inbred lines. Our results suggest that the enhanced ability of salt-tolerant inbred line 'Nongda 1145 ' to combat salt stress occurs via regulation of transcription factors promoting increased antioxidation and lignin biosynthesis, enhanced energy production, and acceleration of protein translation and protein proteolysis.

Key words: Proteome, salt sensitivity, salt stress, salt tolerance, Zea mays.

\section{INTRODUCTION}

Salt stress is a serious constraint factor for plant growth and agricultural productivity on a global scale. Salinity probably affect $6 \%$ of the world's total land and $20 \%$ of cultivated land area (Munns and Tester, 2008). The consequence of plants undergoing salt stress is slow growth and even plant death (Munns and Tester, 2008). Enhancement of crop salt tolerance is consequently an urgent priority (Peng et al., 2009). An understanding of plant response mechanisms under salt stress would provide valuable information for improving stress tolerance.

Plant salinity response is a complex phenomenon involving various physiological, biochemical, and molecular processes (Greenway and Munns, 1980). The injuries induced by salt stress are controlled by a chain of gene expression and proteomic changes. Identification of salt stress-induced protein-level changes is thus a very important approach for understanding the molecular mechanism of response to salt. Plants possessing different

${ }^{1}$ Jiangsu Yanjiang Institute of Agricultural Sciences, Nantong Key Laboratory for Exploitation of Crop Genetic Resources and Molecular Breeding, Rugao, Nantong, Jiangsu, China.

*Corresponding author (417803648@qq.com).

Received: 14 April 2014.

Accepted: 11 August 2014.

doi:10.4067/S0718-58392014000400014 genotypes have different abilities to survive salt stress and rely on various adaptive mechanisms to alleviate salt injury. In a comparative study of two soybean (Glycine $\max$ [L.] Merr.) cultivars under salt stress, a salt-tolerant soybean cultivar exhibited higher ability to scavenge reactive oxygen species, supply energy, produce ethylene, and to enhance photosynthesis (Ma et al., 2012). In wheat (Triticum aestivum L.), V-ATPase, which provides the driving force for compartmentalization of $\mathrm{Na}^{+}$to alleviate ion toxicity, and TCTP, designated as a stress-related protein, were found to be more abundant in the salttolerant cv. Jing-411 than in salt-sensitive CS (a Chinese wheat variety) (Guo et al., 2012).

Maize (Zea mays L.) is an important food, fodder, and cash crop in the world. Globally grown under a wide range of climatic conditions, is sensitive to salinity (Maas and Hoffman, 1977). The root is a vital organ for plants functioning in plant positioning and nutrition uptake, and also the first site for salinity sense, responsible for tolerance to salt stress (Rajaei et al., 2009). Investigating of proteomic expression in different salt resistance maize root would give us insights into molecular mechanism in maize salt tolerance. The common maize inbred line D340, which is widely applied in breeding experiment, is sensitive to salt (Xu, 2010). In contrast, 'Nongda $1145^{\prime}$ ('N1145'), an important inbred line widely used in Chinese maize breeding, was identified as salt tolerant in 
our previous study (Hao et al., 2013). In this study, we conducted a comparative proteomic analysis of ' $\mathrm{N} 1145$ ' and 'D340' roots after exposure to salinity conditions. To serve the purpose of illuminating molecular mechanisms of maize salinity stress response, two-dimensional gel electrophoresis (2-DGE) and matrix-assisted laser desorption/ionization time-of-flight mass spectrometry (MALDI-TOF/TOF-MS) were adopted to analyze protein expression profiles and identify differentially expressed proteins of roots of maize seedlings treated with $\mathrm{NaCl}$.

\section{MATERIALS AND METHODS}

\section{Plant materials and salt treatments}

Seeds of two maize inbred lines, (salt-tolerant inbred line 'N1145' and salt-sensitive inbred line 'D340') were cultivated on plastic trays $(50 \times 50 \times 5 \mathrm{~cm})$ containing quartz sand, seedlings grown in a greenhouse at the Jiangsu Yanjiang Institute of Agricultural Sciences, with the natural daylight condition, temperature $28-35{ }^{\circ} \mathrm{C}$ (day) and $16-20{ }^{\circ} \mathrm{C}$ (night), relative humidity between $65 \%$ and $80 \%$. At first-leaf expansion, seedlings were supplied with nutrient solution. At the two-leaf seedling stage, 15 uniformly seedlings were transplanted to $30 \mathrm{~L}$ plastic containers $(60 \times 40 \times 25 \mathrm{~cm})$ filled with aerated Hoagland nutrient solution. The nutrient solution was renewed every $4 \mathrm{~d}$.

At the three-leaf stage, every maize inbred line seedlings were subjected to two treatments: $0 \mathrm{mM} \mathrm{NaCl}$ (control) in nutrient solution and $150 \mathrm{mM} \mathrm{NaCl}$ in nutrient solution. Each treatment was performed with three replicates. After $3 \mathrm{~d}$ of $\mathrm{NaCl}$ treatment, maize seedling roots were used for the proteomic analysis. Biomass parameters of the maize seedling roots were measured after $7 \mathrm{~d}$ of $\mathrm{NaCl}$ treatment.

\section{Biomass measurement and data analysis}

We measure the fresh weight (FW) of maize shoots after $\mathrm{NaCl}$ treatment for $7 \mathrm{~d}$. After measurement of $\mathrm{FW}$, shoots were maintained in an oven at $105^{\circ} \mathrm{C}$ for $15 \mathrm{~min}$ and then at $80{ }^{\circ} \mathrm{C}$ for $72 \mathrm{~h}$. Shoot dry weight (DW) were then measured. Statistical comparisons were performed by ANOVA followed by the LSD test with a $p<0.05$ significance level using SAS software (SAS Institute, Cary, North Carolina, USA). Fresh weight and DW were calculated as means $\pm \mathrm{SE}(n=15)$.

\section{Two-dimensional electrophoresis}

Root proteins were isolated by phenol extraction according to the procedure of Carpentier et al. (2005). The manual gained from General Electric Healthcare Life Sciences (Little Chalfont, UK) was adopted for two-dimensional gel electrophoresis (2-DGE) of protein sample.

Protein spot images were visualized using silverstaining and scanned at 300 dpi with a UMAX PowerLook 2100XL scanner (Maximum Tech, Taiwan, China). We used Image Master 2D Platinum software version 5.0
(Amersham BioSciences, Little Chalfont, UK) to analyze image. Protein spots which possess good repeatability and significant differences in abundance (changes in volume $\% \geq 1.5$-fold and $p \leq 0.05$ between different treatments) were excised from gels and then stored at $-4^{\circ} \mathrm{C}$ for mass spectrometric analysis.

\section{In-gel digestion and MALDI-TOF/TOF-MS analysis} ABI 4800 proteomics analyzer (Applied Biosystems, Framingham, Massachusetts, USA) was used to identify proteins by matrix-assisted laser desorption/ionization time-of-flight mass spectrometry (MALDI-TOF/TOFMS). The resulting peptide mass lists were searched against the National Center for Biotechnology Information (NCBI, Bethesda, Maryland, USA) maize sequence database (http://www.ncbi.nlm.nih.gov/). The significant matches which the MASCOT probabilistic analysis $(p<$ $0.05)$ determined were accepted.

\section{RESULTS}

Morphological and biomass changes under salt stress To study the two different maize inbred line seedling response to salt stress, seedlings of ' $\mathrm{N} 1145$ ' and 'D340' were treated at the three-leaf stage with $\mathrm{NaCl}$ for $7 \mathrm{~d}$. After salt treatment, 'D340' plants were more wilted than those of 'N1145' and exhibited obvious chlorosis; in addition, shoot FW and DW were decreased more in 'D340' than in 'N1145' (Figure 1). Under salt stress, FW of 'N1145'

A

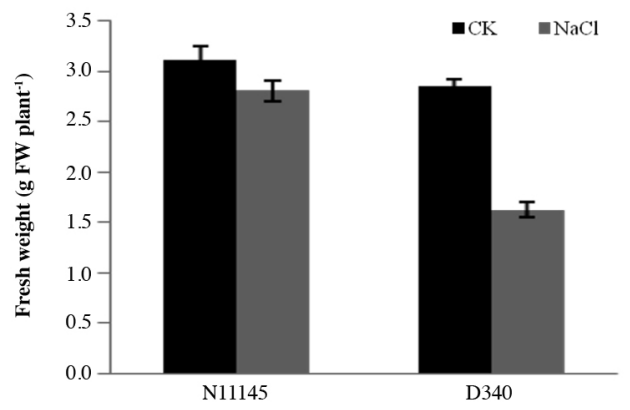

B

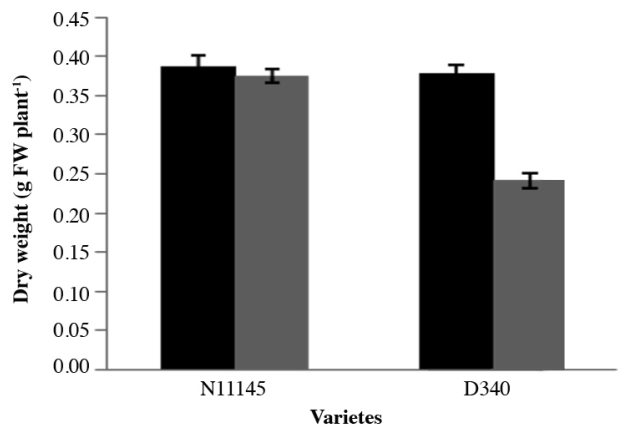

Figure 1. Effects of salt stress on (A) fresh weight and (B) dry weight of shoots of two maize inbred lines (salt-tolerant N1145 and saltsensitive D340) exposed to $\mathrm{NaCl}$ for $7 \mathrm{~d}$. CK corresponds to control (no $\mathrm{NaCl}$ ) conditions. Vertical bars represent means $\pm \mathrm{SE}$ of results from five independent experiments. 
shoots was only reduced to $90.01 \%$ of the non-salt control, a non-significant difference; on the other hand, FW of 'D340' under salt stress was significantly reduced to $56.95 \%$ of the control. Similarly, DW of 'N1145' shoots under salt treatment was $97.07 \%$ of the non-salt control, a non-significant difference, but was significantly reduced to $63.84 \%$ in 'D340' under salt stress. These results clearly demonstrate that salt stress reduced shoot biomass and inhibited plant growth in 'D340' seedlings, but had no discernible effect on 'N1145'.

\section{NaCl-Responsive proteins}

We conducted a proteomic study to further analyze maize response to salt stress, and observed a consistent protein expression pattern on the resulting gels. There were 72 proteins showed more than 1.5-fold differences ( $p<$ 0.05 ) in expression values under salt stress compared with the control (Figure 2). In total, 26 and 46 proteins were differentially expressed in 'N1145' and 'D340', respectively (Figure 2). In 'N1145' under salt stress, the abundance of 14 proteins was increased and 12 proteins decreased. In 'D340', 26 proteins increased in relative abundance and 20 proteins decreased. The majority of changed spots could be easily observed in 'D340'.

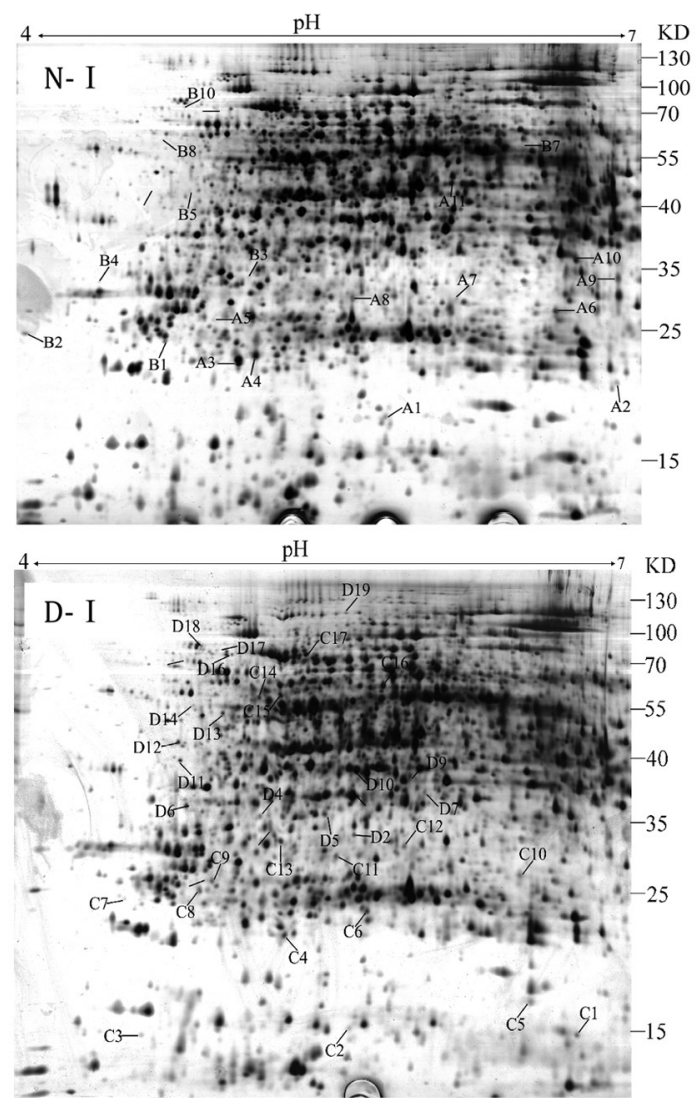

\section{Protein identification}

The MALDI-TOF/TOF-MS was applied for analyzing the 72 differentially expressed proteins. Fifty-seven of 72 proteins matched very well; 15 did not. Table 1 shows the results of database search. A great amount of the matched proteins have functional annotations in the databases (NCBI or Protein Information Resource, PIR), whereas 10 proteins (spots B6, B8, C1, C2, C8, C16, D1, D3, D4, and D11) did not be annotated described as unknown or hypothetical protein (Table 1).

For the latter, the resulting peptide mass lists were searched via Mascot Server (version 2.3.0, Matrix Science, Boston, Massachusetts, USA) against release 57.15 of the Swiss-Prot, Universal Protein Resource (UniProt) Knowledgebase (UniProtKB) protein database (UniProt Consortium European Bioinformatics Institute [EBI], Hinxton, UK/Swiss Institute of Bioinformatics [SIB], Geneva, Switzerland/Protein Information Resource [PIR], Georgetown, USA) constrained to Viridiplantae taxonomy. Only homolog of D11 was found, no homologs of other nine spots were found, indicating that these salt stress-responsive proteins may be unique to maize; their functions thus remain unknown.

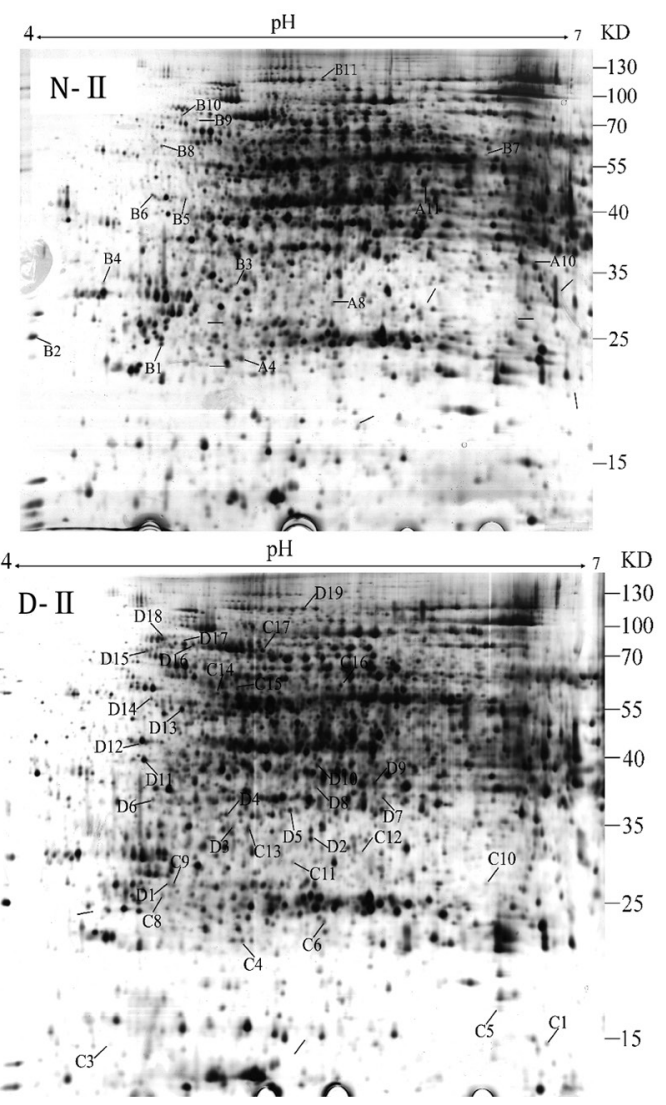

Figure 2. Two-dimensional electrophoretic images of root protein profiles of maize inbred lines N1145 (N) and D340 (D). Protein samples were treated with $0 \mathrm{mM}$ (I) or $150 \mathrm{mM}$ (II) NaCl solutions. Spots labeled with A and C correspond to salt stress-induced down-regulation in the roots of 'N1145' and 'D340', respectively, while B and D indicate up-regulation. 
Table 1. Differentially expressed proteins identified in maize seedling roots of N1145 and D340 inbred lines under salt stress.

\begin{tabular}{|c|c|c|c|c|c|c|c|c|c|}
\hline Spot & Accession nr & Protein name & $\begin{array}{c}\text { Protein } \\
\text { score }\end{array}$ & $\mathrm{TMr} / \mathrm{EMr}$ & TpI/EpI & $\begin{array}{c}\text { Fold } \\
\text { change }^{\mathrm{a}}\end{array}$ & MP & UP & $\mathrm{C}$ \\
\hline A1 & gil413921028 & Hypothetical protein ZEA MMB73_143593 [Zea mays] & 146 & $17171 / 24406$ & $5.70 / 9.27$ & Loss & 9 & 20 & 21 \\
\hline A2 & gil413923108 & Putative glycolipid transfer protein (GLTP) family protein isoform 3 [Zea mays] & 121 & $18928 / 22757$ & $6.59 / 6.52$ & Loss & 7 & 25 & 40 \\
\hline A3 & gil195623546 & Adenine phosphoribosyltransferase 1 [Zea mays] & 389 & $21197 / 19555$ & $5.17 / 5.14$ & -1.61 & 11 & 44 & 66 \\
\hline A4 & gil195607172 & 1,2-Dihydroxy-3-keto-5-methylthiopentene dioxygenase 2 [Zea mays] & 253 & $21648 / 23623$ & $5.24 / 5.07$ & -1.64 & 9 & 20 & 34 \\
\hline A5 & gil413953523 & Catalytic/hydrolase isoform 2 [Zea mays] & 126 & $25584 / 27487$ & $5.08 / 4.91$ & Loss & 4 & 25 & 20 \\
\hline A6 & gil226506734 & Ribose-5-phosphate isomerase [Zea mays] & 228 & $25646 / 26900$ & $6.38 / 5.98$ & Loss & 6 & 14 & 29 \\
\hline A7 & gil413945155 & Putative $O$-glycosyl hydrolase superfamily protein [Zea mays] & 123 & $27686 / 33078$ & $5.99 / 5.46$ & Loss & 4 & 14 & 18 \\
\hline A8 & gil413923017 & Nuclear cap-binding protein subunit 2 [Zea mays] & 195 & $28298 / 28266$ & $5.61 / 5.54$ & -1.93 & 6 & 12 & 24 \\
\hline A9 & gil413922097 & Retrotransposon protein Ty1-copia subclass [Zea mays] & 247 & $31643 / 28924$ & $6.64 / 6.31$ & Loss & 5 & 21 & 23 \\
\hline A 10 & gil413924993 & Putative MADS-box transcription factor family protein [Zea mays] & 94 & $33340 / 29705$ & $6.54 / 9.52$ & -4.14 & 8 & 20 & 22 \\
\hline A11 & gil195626878 & Methylthioribose kinase [Zea mays] & 303 & $40955 / 48366$ & $5.98 / 5.83$ & -1.56 & 11 & 29 & 30 \\
\hline B1 & gil226530826 & Proteasome subunit alpha type 5 [Zea mays] & 174 & $22600 / 26103$ & $4.88 / 4.72$ & 1.76 & 10 & 33 & 45 \\
\hline B2 & gil162462924 & Chitinase chem5 precursor [Zea mays] & 80 & $22719 / 30442$ & $4.29 / 4.10$ & 1.58 & 3 & 13 & 8 \\
\hline B3 & gil413942982 & Caffeoyl-CoA 3-O-methyltransferase 1 [Zea mays] & 103 & $30921 / 27194$ & $5.21 / 5.22$ & 1.67 & 2 & 9 & 13 \\
\hline B4 & gil226503896 & Elongation factor 1-delta 1 [Zea mays] & 118 & $29383 / 24951$ & $4.60 / 4.39$ & 4.47 & 6 & 29 & 27 \\
\hline B5 & gil293335955 & Uncharacterized protein LOC100382168 precursor [Zea mays] & 189 & $39507 / 53269$ & $4.98 / 7.91$ & 2.50 & 6 & 6 & 14 \\
\hline B6 & gil194701538 & Unknown [Zea mays] & 39 & $39544 / 21166$ & $4.83 / 9.07$ & New & 2 & & 10 \\
\hline B7 & gil3342800 & Putative cytosolic 6-phosphogluconate dehydrogenase [Zea mays] & 126 & $53537 / 53307$ & $6.29 / 5.92$ & 2.49 & 8 & 8 & 20 \\
\hline B8 & gil293333779 & Uncharacterized protein LOC100382017 [Zea mays] & 150 & $56013 / 60020$ & $4.88 / 4.83$ & 4.50 & 7 & 11 & 17 \\
\hline B9 & gil413920734 & Protein disulfide isomerase 1 [Zea mays] & 83 & $66617 / 40588$ & $4.92 / 5.21$ & 2.24 & 5 & 8 & 12 \\
\hline B10 & gil238928460 & Cytosolic orthophosphate dikinase [Zea mays] & 219 & $100927 / 96354$ & $5.53 / 5.20$ & 3.26 & 17 & 10 & 17 \\
\hline $\mathrm{C} 1$ & gil226531418 & Uncharacterized protein LOC100275940 [Zea mays] & 256 & $13922 / 15184$ & $6.47 / 6.75$ & -1.52 & 7 & 37 & 63 \\
\hline $\mathrm{C} 2$ & gil195605262 & Hypothetical protein [Zea mays] & 88 & $13788 / 11495$ & $4.75 / 4.74$ & Loss & 4 & 16 & 38 \\
\hline $\mathrm{C} 3$ & gil162464130 & Eukaryotic translation initiation factor 5A [Zea mays] & 145 & $14406 / 17714$ & $5.57 / 5.61$ & -1.95 & 5 & 23 & 28 \\
\hline $\mathrm{C} 4$ & gil413954658 & Proteasome subunit beta type [Zea mays] & 224 & $20520 / 23166$ & $5.33 / 5.28$ & -1.57 & 8 & 17 & 34 \\
\hline $\mathrm{C} 5$ & gil226501178 & Flavoprotein wrbA [Zea mays] & 156 & $20216 / 22497$ & $6.11 / 5.95$ & -2.17 & 3 & 25 & 20 \\
\hline C6 & gil226532014 & Proteasome component2 [Zea mays] & 170 & $21801 / 23240$ & $5.62 / 5.60$ & -1.53 & 9 & 45 & 43 \\
\hline $\mathrm{C} 7$ & gil226532014 & Proteasome component2 [Zea mays] & 83 & $22540 / 23240$ & $4.67 / 5.60$ & Loss & 6 & 13 & 28 \\
\hline $\mathrm{C} 8$ & gil226505188 & Hypothetical protein [Zea mays] & 193 & $23676 / 27987$ & $4.98 / 4.82$ & -1.92 & 4 & 21 & 22 \\
\hline C9 & gil226491378 & Chalcone flavanone isomerase 1 [Zea mays] & 251 & $25061 / 24065$ & $5.03 / 4.99$ & -1.78 & 5 & 30 & 25 \\
\hline $\mathrm{C} 10$ & gil195641684 & Proteasome subunit alpha type 6 [Zea mays] & 256 & $25213 / 27584$ & $6.29 / 6.11$ & -1.88 & 9 & 31 & 44 \\
\hline $\mathrm{C} 11$ & gil195643974 & Soluble inorganic pyrophosphatase [Zea mays] & 255 & $28195 / 24276$ & $5.53 / 5.31$ & -1.67 & 7 & 18 & 34 \\
\hline $\mathrm{C} 12$ & gil226495791 & Soluble inorganic pyrophosphatase [Zea mays] & 104 & $28958 / 25686$ & $5.77 / 5.58$ & -2.07 & 5 & 31 & 27 \\
\hline $\mathrm{C} 13$ & gil195620650 & Eukaryotic translation initiation factor 3 subunit 5 [Zea mays] & 386 & $30474 / 31501$ & $5.31 / 5.17$ & -2.14 & 9 & 36 & 37 \\
\hline $\mathrm{C} 14$ & gil226493589 & ATP synthase beta chain [Zea mays] & 271 & $53990 / 59057$ & $5.19 / 5.90$ & -1.59 & 14 & 8 & 37 \\
\hline $\mathrm{C} 15$ & gil226493589 & ATP synthase beta chain [Zea mays] & 465 & $53808 / 59057$ & $5.26 / 5.90$ & -1.91 & 19 & 20 & 46 \\
\hline $\mathrm{C} 16$ & gil414877694 & TPA: hypothetical protein ZEAMMB73_373833 [Zea mays] & 182 & $55867 / 63660$ & $5.65 / 6.08$ & -1.68 & 11 & 18 & 18 \\
\hline $\mathrm{C} 17$ & gil162457903 & Luminal-binding protein 3 precursor [Zea mays] & 313 & $67932 / 73283$ & $5.37 / 5.13$ & -1.64 & 19 & 20 & 28 \\
\hline D1 & gil226508002 & LOC100283026 [Zea mays] & 248 & $24956 / 29044$ & $5.01 / 4.82$ & New & 11 & 14 & 30 \\
\hline D2 & gil413946955 & Phenazine biosynthesis protein [Zea mays] & 387 & $31148 / 31871$ & $5.60 / 5.30$ & 1.64 & 9 & 18 & 31 \\
\hline D3 & gil223946537 & Unknow [Zea mays] & 128 & $32147 / 35255$ & $5.29 / 5.15$ & New & 5 & 19 & 16 \\
\hline D4 & gil226505826 & Uncharacterized protein LOC100279462 [Zea mays] & 213 & $34118 / 33250$ & $5.26 / 5.12$ & 2.08 & 7 & 22 & 33 \\
\hline D5 & gil226508482 & LOC100282555 [Zea mays] & 151 & $35226 / 27949$ & $5.53 / 5.39$ & 4.02 & 4 & 15 & 17 \\
\hline D6 & gil212274981 & 1-Aminocyclopropane-1-carboxylate oxidase 1Acc oxidase [Zea mays] & 96 & $35485 / 35445$ & $4.95 / 4.89$ & 2.68 & 10 & 26 & 25 \\
\hline D7 & gil162458737 & Cysteine synthase precursor [Zea mays] & 143 & $35763 / 34299$ & $5.86 / 5.91$ & 1.62 & 7 & 29 & 30 \\
\hline D8 & gil18202485 & RecName: Full = Malate dehydrogenase, cytoplasmic $[$ Zea mays $]$ & 103 & $36677 / 35909$ & $5.61 / 5.77$ & New & 5 & 22 & 16 \\
\hline D9 & gil33641738 & $O$-methyltransferase [Zea mays] & 191 & $36543 / 39208$ & $5.81 / 5.48$ & 2.11 & 5 & 19 & 17 \\
\hline D10 & gil226531223 & Uncharacterized protein LOC100272868 [Zea mays] & 372 & $38241 / 38392$ & $5.61 / 5.51$ & 1.92 & 9 & 23 & 24 \\
\hline D11 & gil226531223 & Uncharacterized protein LOC100272868 [Zea mays] & 86 & $38206 / 38392$ & $4.90 / 5.51$ & 2.83 & 2 & 9 & 8 \\
\hline D12 & gil195625028 & DNA repair protein RAD23 [Zea mays] & 70 & $39453 / 42164$ & $4.89 / 4.66$ & 2.97 & 4 & 23 & 14 \\
\hline D13 & gil162462441 & Tubulin beta-3 chain [Zea mays] & 183 & $47023 / 50462$ & $5.07 / 4.79$ & 1.98 & 12 & 13 & 23 \\
\hline D14 & gil226493235 & LOC100281701 [Zea mays] & 294 & $50641 / 61419$ & $4.94 / 5.20$ & 4.77 & 11 & 11 & 25 \\
\hline D15 & gil162461230 & Protein disulfide isomerase 2 precursor [Zea mays] & 102 & $65840 / 56876$ & $4.90 / 4.91$ & New & 6 & 11 & 12 \\
\hline D16 & gil413953492 & Putative TCP-1/cpn60 chaperonin family protein isoform 3 [Zea mays] & 292 & $67844 / 61969$ & $5.09 / 5.42$ & 2.45 & 8 & 10 & 16 \\
\hline D17 & gil413926906 & Putative TCP-1/cpn60 chaperonin family protein isoform 2 [Zea mays] & 459 & $67844 / 64610$ & $5.05 / 5.81$ & 2.20 & 13 & 17 & 30 \\
\hline D18 & gil145666464 & Protein disulfide isomerase Zea mays] & 290 & $68645 / 56921$ & $4.97 / 5.01$ & 1.94 & 10 & 26 & 20 \\
\hline D19 & gil238928460 & Cytosolic orthophosphate dikinase [Zea mays] & 308 & $100927 / 96354$ & $5.53 / 5.20$ & 2.27 & 25 & 33 & 24 \\
\hline
\end{tabular}

TMr: Theoretical molecular mass; EMr: experimental molecular mass; TpI: theoretical isoelectric point; EpI: experimental isoelectric point; MP: number of peptides matching the indicated sequence; Up: number of unmatched peaks; C: total percentage of sequence coverage (\%).

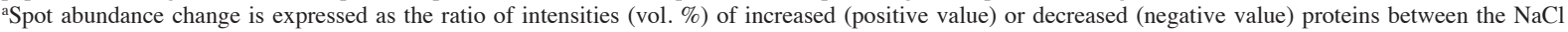
treatment and the control. A paired Student's t-test was used to identify significant changes $(p<0.05)$ between protein spots from NaCl-treated and control seedlings. 'Lost' refers to protein spots that were undetectable after $\mathrm{NaCl}$ treatment; 'New' indicates protein spots not detectable in the control.

Gene ontology analysis of $\mathrm{NaCl}$-responsive proteins

The functional classifications of salt-regulated proteins were carried out according to the gene ontology biological process (GOBP) and gene ontology molecular function (GOMF) sections in QuickGO database (European Molecular Biology Laboratory [EMBL]-
European Bioinformatics Institute [EBI], Hinxton, UK). Salt-regulated proteins distributed on various biological processes. In salt-regulated proteins of 'N1145' (Figure $3 \mathrm{~A})$, carbohydrate metabolic were the highest proportion $(47.4 \%)$ of GOBP, which included carbohydrate hydrolysis, pentose phosphate pathway (PPP), and 
A

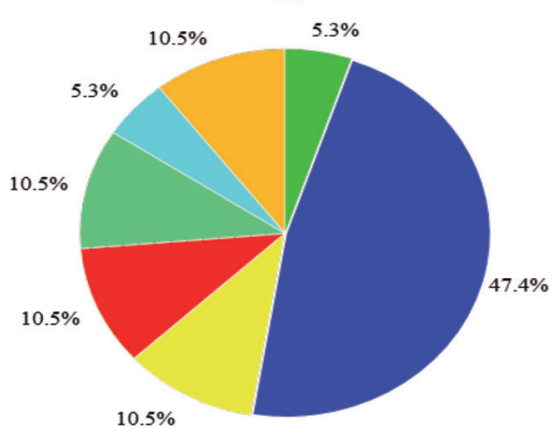

$10.5 \%$

Eedox homeostasis

- Carbohydrate metabolism

$\square$ Nucleotide metabolism

- Amino acid and nitrogen metabolism

- Protein metabolism

- Cell membrane-related proteins

- Transcription and translation-associated proteins

\section{Biological process}

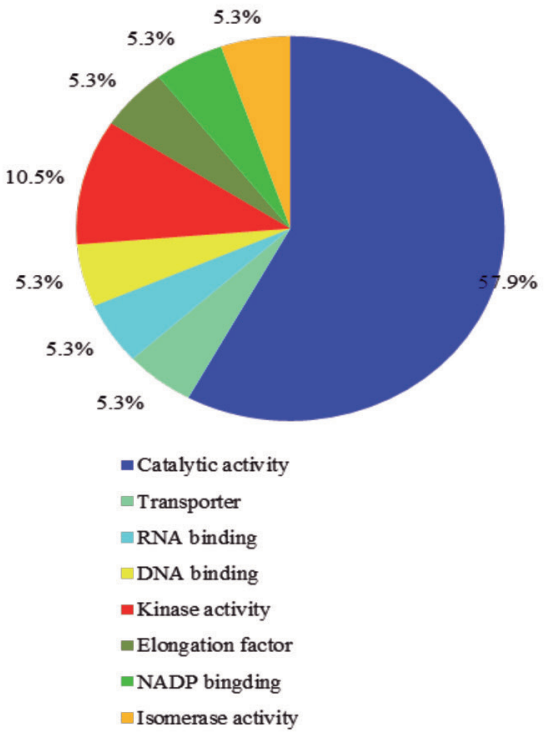

Molecular function

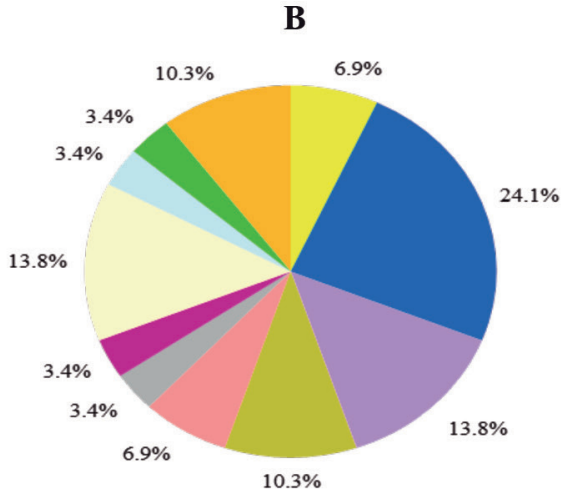

$\square$ Transcription and translation-associated proteins

- Protein metabolism

Redox homeostasis

$\square$ Biosynthesis and secondery metabolites

$\square$ Transport

Response to stress

- Amino acid and nitrogen metabolism

Carbohydrate metabolism

$\square$ Nucleotide repair

Cytoskeleton-associated proteins

$\square$ Other metabolism process

\section{Biological process}

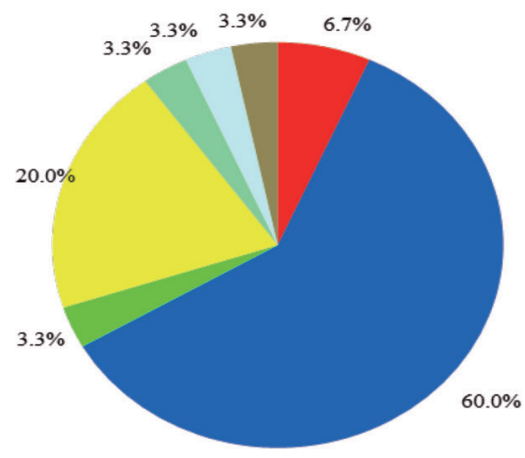

-Iniation factor

- Catalytic activity

$=\mathrm{FMN}$ binding

$\square$ ATP binding

$\square$ DNA binding

$\square$ Cytoskeleton constituent

Kinase activity

Molecular function

Figure 3. Gene ontology (GO) functional classification of salt-responsive proteins in (A) 'N1145' and (B) 'D340' maize seedling roots. Identified annotated proteins are included and presented according to biological process and molecular function. The percentage distributions of the GO terms were calculated using the iProClass GO tool in the Protein Information Resource (PIR) database.

pyruvate processes. Nucleotide metabolism, amino acid and nitrogen metabolism, protein metabolism, and transcription and translation-associated proteins were the second most highly enriched set of GOBP groups. With respect to GOMF categories, enzyme activity (including catalytic activities, kinase activity, and isomerase activity) represented the largest fraction $(73.7 \%)$ followed by binding activity (including RNA, DNA, and NADP) $(15.8 \%)$. The salt stress-regulated proteins in 'D340'
(Figure 3B), the highest proportion of GOBP category was protein metabolic processes $(24.1 \%)$. Redox homeostasis $(13.8 \%)$ and carbohydrate metabolism $(13.8 \%)$ were the next highest proportion of GOBP categories. The top two GOMF categories were catalysis enzyme activity $(60.0 \%)$ and ATP-binding activity (20.0\%). According to this analysis, $\mathrm{NaCl}$-responsive proteins in 'N1145' and 'D340' had approximately similar functional distributions. 


\section{DISCUSSION}

\section{Antioxidative reactions}

The oxidative damage of plant is triggered by salt stress (Abogadallah et al., 2010). To combat salt stress, plants adopt complex antioxidant defense system inducing antioxidant enzymes and antioxidants. In this study, three identified protein spots were related to antioxidative defense system in maize roots under salt stress. In particular, abundances of protein spots B9 in inbred line N1145 and D15 and D18 in 'D340' were significantly ( $p<$ 0.05 ) up-regulated in seedling roots of the two maize lines under salt stress. We identified the three protein spots as protein disulfide isomerase (PDI), which involves in the pathway of thioredoxin redox and severs as a important part of antioxidative defense system (Wan and Liu, 2008). The antioxidative defense system can reduce salt stressinduced reactive oxygen species (ROS) concentrations and protect the plant from oxidative stress. The antioxidant PDI has also been found to be impacted by salt stress in soybean. Under salt stress, PDI was shown to be upregulated in leaves of the salt-tolerant soybean genotype 'Lee' but down-regulated in salt-sensitive 'Jackson' (Ma et al., 2012). These results indicate that maize plants can better acclimate to salt by stimulating their antioxidative defense system.

\section{Secondary metabolism}

$O$-methyltransferase is a key enzyme in the biosynthesis of lignins, flavonoids, and other secondary metabolites. Caffeoyl-CoA3-O-methyltransferase, catalyzing the methylation of caffeoyl-CoA into feruloyl-CoA, is a lignin biosynthesis precursor (Whetten and Sederoff, 1995). In this study, protein spots B3 (identified as caffeoylCoA 3-O-methyltransferase) and $\mathrm{D} 9$ (corresponding to $O$-methyltransferase) were significantly up-regulated under salt stress in seedling roots of 'N1145' and 'D340', respectively. This observed increase in $O$-methyltransferase protein suggests that lignin biosynthesis is affected by salt. Touzet et al. (1996) have reported increased amounts of $O$-methyltransferase in drought-affected maize plants, and induction of $O$-methyltransferase in maize leaves under water deficit has also been observed (Riccardi et al., 1998). This enzyme has also been found to be involved in response to infection due to pathogen attack (Maury et al., 1999). These results indicate that lignin biosynthesis plays a crucial role in plant response to adverse environmental conditions. In this study, $O$-methyltransferase was upregulated in both salt-tolerant and salt-sensitive maize inbred lines. Our observations suggest that this enzyme may increase lignin amounts by lignification, promoting thereby strong seedling growth and resistance to salt stress-induced injuries.

Flavonoids are involved in a vast array of plant biological functions associated with defenses against biotic and abiotic stress (Harborne and Williams, 2000;
Winkel-Shirley, 2002; Taylor and Grotewold, 2005; Lepiniec et al., 2006; Pourcel et al., 2007; Xu et al., 2008). The abundance of one key flavonoid pathway enzyme, chalcone isomerase ( $\mathrm{CHI}$; protein spot $\mathrm{C} 9$ ), was downregulated under salinity stress in our experiment in saltsensitive 'D340', but remained unchanged $(p>0.05)$ in seedling roots of 'N1145'. The decrease in CHI activity led to decreased flavonoid levels in the salt-sensitive inbred line. The contrasting CHI expression patterns observed in seedling roots of 'D340' and 'N1145' may be partially responsible for the different salinity tolerances of these two maize inbred lines.

\section{Carbohydrate metabolism}

Proteins associated with two different carbohydrate metabolic pathways were uncovered in this study. Two proteins (spots A6 and B7) were identified as enzymes participating in the PPP. Some enzymes of PPP play an important role in the stress-induced production of NADPH in animals and plants (Meral et al., 2009). B7 corresponded to 6-phosphogluconate dehydrogenase (6PGDH), producing a NADPH molecule accompanied by catalytic reaction, is a rate-limiting enzyme in the oxidative PPP (Alam et al., 2011). In our study, it was up-regulated under salinity treatment only in the salttolerant inbred line, suggesting that the enhancement of the oxidative PPP occurred only in that line. In rice, 6PGDH protein and transcript levels are up-regulated in response to salt stress (Huang et al., 2003; Hauschild and von Schaewen, 2003; Hou et al., 2007; Witzel et al., 2010). Spot A6 was identified as ribose-5-phosphate isomerase (R5PI), an enzyme in the non-oxidative PPP that catalyzes the conversion of ribulose-5-phosphate to ribose-5-phosphate. In this study, it was down-regulated under salt stress only in the tolerant inbred line. The oxidative PPP provides reduced NADPH, which acts as an electron donor to cytochromes and other electron carriers during biosynthesis of energy (ATP), and thus ensures the availability of ATP for synthesis of plant stress-responsive proteins. 6PGDH and R5PI activities were respectively up- and down-regulated in N1145 seedling roots during different phases of the PPP. We infer from these results that the tolerant inbred line can enhance its energy utilization efficiency by increasing 6PGDH activity and decrease unnecessary energy consumption by down-regulating R5PI. 6PGDH genes may be appropriate indexes for enhancing plant salt tolerance.

The other protein related to carbohydrate metabolism, spot D8, was identified as cytoplasmic malate dehydrogenase (cyMDH). In our study, this enzyme was up-regulated under salt treatment only in the sensitive inbred line. It is up-regulated in wheat under drought conditions and in cucumber under salt stress (Du et al., 2010; Ge et al., 2012). Yao et al. (2011) have reported that overexpression of cyMDH can improve growth, increase cold and salt tolerance in apple and tomato. 
To summarize, the TCA cycle was unaffected by salt stress in the salt-tolerant inbred line, and this insensitivity to salinity was coupled with up-regulation of the energyproducing phase of the PPP to combat stress injury. In the salt-sensitive inbred line, in contrast, salt stress upregulated MDH activity but not the oxidative PPP phase. We infer from these results that the salt-tolerant maize line produced more energy by the PPP and the TCA pathway, giving it more tolerance compared with the salt-sensitive inbred line.

\section{Protein metabolism}

We identified 10 salt stress-responsive proteins involved in protein metabolism, which can be categorized into three groups (Figure 2). There were three protein spots with the function of protein biosynthesis in the first group, one translation elongation factor (spot B4) and two translation initiation factors (spots $\mathrm{C} 3$ and $\mathrm{C} 13$ ). The salt stress only up-regulated translation elongation factor (spot B4) in tolerant maize inbred line (Table 1). Nam et al. (2012) have reported that two translation elongation factors are up-regulated under salt stress in rice. Under salt stress, the translation initiation factors (spots $\mathrm{C} 3$ and $\mathrm{C} 13$ ) were significantly $(p<0.05)$ down-regulated in sensitive maize inbred line, but were unaffected in the tolerant line. The initiation and elongation of peptide chains rely on these proteins (Wan and Liu, 2008). Previous studies have suggested that translation initiation and elongation factors play key regulatory roles in plant growth and stress responses (Sahi et al., 2006; Ma et al., 2010). Our results suggest that the biosynthesis of these proteins under salt stress was increased in the salt-tolerant line, but was decreased in the salt-sensitive line.

The second group was related to protein folding and assembly, including: refolding protein (spot D14), TCP-1 chaperonin isoform 3 (spot D16) and TCP-1 chaperonin isoform 2 (spot D17). Under salt stress, all three proteins were significantly $(p<0.05)$ up-regulated in the sensitive maize inbred line but not in the tolerant inbred line. Refolding protein and chaperonin are responsible for restoration of the biological activity of unfolded and misfolded proteins, taking a protective role combat with stress (Wang et al., 2004). In our study, the exclusive up-regulation of refolding protein and chaperonin in the sensitive maize inbred line under salt stress led to the following conclusion: while the sensitive inbred line suffered severe damage, the tolerant inbred line underwent only mild injury, eliminating the need for this repair mechanism.

The third group was involved in the protein proteolysis pathway including six proteins (spots B1, B5, C4, C6, $\mathrm{C} 7$, and $\mathrm{C} 10$ ). These proteins, identified as proteasomes, were up-regulated under salt stress in salt-tolerant 'N1145' and down-regulated in salt-sensitive 'D340'. In previous studies, the involvement of proteolytic pathway proteins such as proteases in salt tolerance has been inferred on the basis of increases in transcript levels or enzyme activities (Manivannan et al., 2008; Li et al., 2009; Zhou et al., 2010). Our results indicate that a plant might increase its salt tolerance by enhancing its protein proteolysis capacity.

\section{Energy and transport}

Ion toxicity is one of the most severely damaging consequences of salt stress. To cope with ion imbalance, plants have developed the mechanism of ion compartmentalization to maintain ion homeostasis (Tester and Davenport, 2003). ATPases supply driving force for ion compartmentalization by ATP synthesis and/or hydrolysis. We identified two mitochondrial ATP synthase proteins (spots C14 and C15) in this study. Both proteins were down-regulated in seedling roots of 'D340', but were not detected in 'N1145'. The mitochondrial ATP synthase complex mediates transportation of $\mathrm{H}^{+}$ions from the outer compartment to the inner mitochondrial matrix via its channels, producing a proton-motive force that drives the transport of all other ions and metabolites (Zhu, 2003). Because the ATP protein was only decreased in the sensitive inbred line, we conclude that salt stress impacted the ion compartmentalization ability of the sensitive genotype but that ion homeostasis of the tolerant inbred line was unaffected.

\section{Transcription}

The pathway of transcriptional factors regulation is crucial for plants responding to stress (Jiang et al., 2007). In this study, we detected three transcriptionalassociated proteins: nuclear cap-binding protein (spot A8), retrotransposon protein (spot A9) and putative MADS-box transcription factor (A10). Cap-binding protein functions in pre-mRNA splicing, a fundamental step in both constitutive and regulated gene expression and often related to stress response (Glisovic et al., 2008). Retrotransposon protein regulates the transposition of DNA. MADS-box transcription factors are key regulators of several plant development processes (Parenicová et al., 2003). All three proteins were down-regulated in seedling roots of salt-tolerant ' $\mathrm{N} 1145$ ', but were unaffected in saltsensitive 'D340'. This result suggests that the salt-tolerant maize inbred line may enhance its adaptation to salinity by regulating transcription to control gene expression. Spot D12, DNA repair protein, was up-regulated in seedling roots of 'D340' but undetected in 'N1145'. The DNA of salt-sensitive maize inbred line 'D340' may suffer serious damage under salinity, leading to high abundance of this protein; the degree of DNA injury in 'N1145', on the other hand, was insufficient to elevate abundance of the DNA repair protein.

In this work, a proteomics in response to salt stress in maize seedlings was researched; several metabolism pathways were induced by salt stress. For clarifying precise mechanism in response to salinity for maize, further studies will be conducted. Some targeted proteins should 
be chosen for studying the transcriptional expression, and the specific mutants should be used to obtain additional evidence. Meanwhile, the correlation between different metabolism pathways should be revealed.

\section{CONCLUSIONS}

To investigate global protein changes under salinity, we carried out a comparative proteomic analysis of two maize inbred line (salt-tolerant 'N1145' and salt-sensitive 'D340') using $\mathrm{NaCl}$ for salt treatment. We successfully identified 57 protein spots as differently expressed in salttreated maize seedling roots. These proteins involved in antioxidant system, secondary metabolite biosynthesis, carbohydrate metabolism, protein translation, protein refolding, proteolysis, energy metabolism and transcriptional regulation. A few proteins may be applied to prediction of salt tolerance in maize. Additional research is needed to confirm their presence in a wider range of germplasm resources.

\section{ACKNOWLEDGEMENTS}

We gratefully acknowledge funding from projects supported by the Jiangsu Academy of Independent Innovation Funds of China (CX(11)4059) and the Nantong City Science and Technology Funds of China (AS2011017 and HL2013017).

\section{LITERATURE CITED}

Abogadallah, G.M., M.M. Serag, and W.P. Quick. 2010. Fine and coarse regulation of reactive oxygen species in the salt tolerant mutants of barnyard grass and their wild-type parents under salt stress. Physiologia Plantarum 138:60-73.

Alam, I., S.A. Sharmin, K.-H. Kim, Y.-G. Kim, J.J. Lee, J.D. Bahk, et al. 2011. Comparative proteomic approach to identify proteins involved in flooding combined with salinity stress in soybean. Plant and Soil 346:45-62.

Carpentier, S.C., E. Witters, K. Laukens, P. Deckers, R. Swennen, and B. Panis. 2005. Preparation of protein extracts from recalcitrant plant tissues: An evaluation of different methods for two-dimensional gel electrophoresis analysis. Proteomics 5:24972507.

Du, C.-X., H.-F. Fan, S.-R. Guo, T. Tezuka, and J. Li. 2010. Proteomic analysis of cucumber seedling roots subjected to salt stress. Phytochemistry 71:1450-1459.

Ge, P., C. Ma, S. Wang, L. Gao, X. Li, G. Guo, et al. 2012. Comparative proteomic analysis of grain development in two spring wheat varieties under drought stress. Analytical and Bioanalytical Chemistry 402:1297-1313.

Glisovic, T., J.L. Bachorik, J. Yong, and G. Dreyfuss. 2008. RNAbinding proteins and post-transcriptional gene regulation. FEBS Letters 582:1977-1986.

Greenway, H., and R. Munns. 1980. Mechanisms of salt tolerance in nonhalophytes. Annual Review of Plant Physiology 31:149-190.

Guo, G., P. Ge, C. Ma, X. Li, D. Lv, S. Wang, et al. 2012. Comparative proteomic analysis of salt response proteins in seedling roots of two wheat varieties. Journal of Proteomics 75:1867-1885.

Hao, D.R., Y.J. Cheng, C.W. Xu, Y.X. Mao, C.J. Peng, and L. Xue. 2013. Screening of maize germplasms for salt tolerance and evaluation of population genetic structure. Journal of Plant Genetic Resource (in Chinese) 14:1153-1160.
Harborne, J.B., and C.A. Williams. 2000. Advances in flavonoid research since 1992. Phytochemistry 55:481-504.

Hauschild, R., and A. von Schaewen. 2003. Differential regulation of glucose-6-phosphate dehydrogenase isoenzyme activities in potato. Plant Physiology 133:47-62.

Hou, F.Y., J. Huang, S.L. Yu, and H.S. Zhang. 2007. The 6-phosphogluconate dehydrogenase genes are responsive to abiotic stresses in rice. Journal of Integrative Plant Biology 49:655-663

Huang, J., H. Zhang, J. Wang, and J. Yang. 2003. Molecular cloning and characterization of rice 6-phosphogluconate dehydrogenase gene that is up-regulated by salt stressa. Molecular Biology Reports 30:223-227.

Jiang, Y., B. Yang, N.S. Harris, and M.K. Deyholos. 2007. Comparative proteomic analysis of $\mathrm{NaCl}$ stress-responsive proteins in Arabidopsis roots. Journal of Experimental Botany 58:3591-3607.

Lepiniec, L., I. Debeaujon, J.-M. Routaboul, A. Baudry, L. Pourcel, N. Nesi, et al. 2006. Genetics and biochemistry of seed flavonoids. Annual Review of Plant Biology 57:405-430.

Li, H., Y. Wang, J. Jiang, G. Liu, C. Gao, and C. Yang. 2009. Identification of genes responsive to salt stress on Tamarix hispida roots. Gene 433:65-71.

Ma, F., Z. Liu, T.W. Wang, M.T. Hopkins, C.A. Peterson, and J.E. Thompson. 2010. Arabidopsis eIF5A3 influences growth and the response to osmotic and nutrient stress. Plant, Cell and Environment 33:1682-1696.

Ma, H., L. Song, Y. Shu, S. Wang, J. Niu, Z. Wang, et al. 2012. Comparative proteomic analysis of seedling leaves of different salt tolerant soybean genotypes. Journal of Proteomics 75:15291546.

Maas, E., and G. Hoffman. 1977. Crop salt tolerance-current assessment. Journal of the Irrigation and Drainage Division 103:115-134.

Manivannan, P., C.A. Jaleel, B. Sankar, A. Kishorekumar, P. Murali, R. Somasundaram, et al. 2008. Mineral uptake and biochemical changes in Helianthus annииs under treatment with different sodium salts. Colloids and Surfaces B: Biointerfaces 62:58-63.

Maury, S., P. Geoffroy, and M. Legrand. 1999. Tobacco $O$-methyltransferases involved in phenylpropanoid metabolism. The different caffeoyl-coenzyme A/5-hydroxyferuloyl-coenzyme A 3/5-O-methyltransferase and caffeic acid/5-hydroxyferulic acid 3/5-O-methyltransferase classes have distinct substrate specificities and expression patterns. Plant Physiology 121:215224.

Meral, T.O., M. Gad, S. Luhua, K. James, S. Ahmet, K. Shai, et al. 2009. Thiamin confers enhanced tolerance to oxidative stress in arabidopsis. Plant Physiology 151:421-432.

Munns, R., and M. Tester. 2008. Mechanisms of salinity tolerance. Annual Review of Plant Biology 59:651-681.

Nam, M.H., S.M. Huh, K.M. Kim, W.J. Park, J.B. Seo, K. Cho, et al. 2012. Comparative proteomic analysis of early salt stressresponsive proteins in roots of SnRK2 transgenic rice. Proteome Science 10:2-19.

Parenicová, L., S. de Folter, M. Kieffer, D.S. Horner, C. Favalli, J. Busscher, et al. 2003. Molecular and phylogenetic analyses of the complete MADS-box transcription factor family in Arabidopsis new openings to the MADS world. The Plant Cell Online 15:1538-1551

Peng, Z., M. Wang, F. Li, H. Lv, C. Li, and G. Xia. 2009. A proteomic study of the response to salinity and drought stress in an introgression strain of bread wheat. Molecular and Cellular Proteomics 8:2676-2686.

Pourcel, L., J.-M. Routaboul, V. Cheynier, L. Lepiniec, and I. Debeaujon. 2007. Flavonoid oxidation in plants: from biochemical properties to physiological functions. Trends in Plant Science 12:29-36.

Rajaei, S., V. Niknam, S. Seyedi, H. Ebrahimzadeh, and K. Razavi. 2009. Contractile roots are the most sensitive organ in Crocus sativus to salt stress. Biologia Plantarum 53:523-529. 
Riccardi, F., P. Gazeau, D. de Vienne, and M. Zivy. 1998. Protein changes in response to progressive water deficit in maize quantitative variation and polypeptide identification. Plant Physiology 117:1253-1263.

Sahi, C., A. Singh, E. Blumwald, and A. Grover. 2006. Beyond osmolytes and transporters: Novel plant salt-stress tolerancerelated genes from transcriptional profiling data. Physiologia Plantarum 127:1-9.

Taylor, L.P., and E. Grotewold. 2005. Flavonoids as developmental regulators. Current Opinion in Plant Biology 8:317-323.

Tester, M., and R. Davenport. 2003. $\mathrm{Na}^{+}$tolerance and $\mathrm{Na}^{+}$transport in higher plants. Annals of Botany 91:503-527.

Touzet, P., F. Riccardi, C. Morin, C. Damerval, J.-C. Huet, J.-C. Pernollet, et al. 1996. The maize two-dimensional gel protein database: Towards an integrated genome analysis program. Theoretical and Applied Genetics 93:997-1005.

Wan, X.-Y., and J.-Y. Liu. 2008. Comparative proteomics analysis reveals an intimate protein network provoked by hydrogen peroxide stress in rice seedling leaves. Molecular and Cellular Proteomics 7:1469-1488.

Wang, W., B. Vinocur, O. Shoseyov, and A. Altman. 2004. Role of plant heat-shock proteins and molecular chaperones in the abiotic stress response. Trends in Plant Science 9:244-252.

Whetten, R., and R. Sederoff. 1995. Lignin biosynthesis. The Plant Cell 7:1001.
Winkel-Shirley, B. 2002. Biosynthesis of flavonoids and effects of stress. Current Opinion in Plant Biology 5:218-223.

Witzel, K., A. Weidner, G.K. Surabhi, R.K. Varshney, G. Kunze, G.H. Buck-Sorlin, et al. 2010. Comparative analysis of the grain proteome fraction in barley genotypes with contrasting salinity tolerance during germination. Plant, Cell and Environment 33:211-222.

Xu, Y.-X. 2010. TOPSIS method to evaluate the salt-tolerance of silage maize germplasm during the germination and the seedling stage. Rain Fed Crops (in Chinese) 30:101-104.

Xu, C., J.H. Sullivan, W.M. Garrett, T.J. Caperna, and S. Natarajan. 2008. Impact of solar ultraviolet-B on the proteome in soybean lines differing in flavonoid contents. Phytochemistry 69:38-48.

Yao, Y.-X., Q.-L. Dong, H. Zhai, C.-X. You, and Y.-J. Hao. 2011. The functions of an apple cytosolic malate dehydrogenase gene in growth and tolerance to cold and salt stresses. Plant Physiology and Biochemistry 49:257-264.

Zhou, G.-A., R.-Z. Chang, and L.-J. Qiu. 2010. Overexpression of soybean ubiquitin-conjugating enzyme gene GmUBC2 confers enhanced drought and salt tolerance through modulating abiotic stress-responsive gene expression in Arabidopsis. Plant Molecular Biology 72:357-367.

Zhu, J.-K. 2003. Regulation of ion homeostasis under salt stress. Current Opinion in Plant Biology 6:441-445. 